\title{
Major Increase in Microbiota-Dependent Proatherogenic Metabolite TMAO One Year After Bariatric Surgery
}

\author{
Marius Trøseid, MD, PhD, ${ }^{1-4}$ Johannes R. Hov, MD, $\mathrm{PhD}^{2-6}$ Torunn Kristin Nestvold, MD, PhD?, \\ Hanne Thoresen, MD, Rolf K. Berge, $\mathrm{PhD}^{9,10}$ \\ Asbjørn Svardal, $\mathrm{PhD}^{9}$, and Knut Tore Lappegård, $\mathrm{MD}, \mathrm{PhD}^{11,12}$
}

\begin{abstract}
Background: Trimethylamine- $N$-oxide (TMAO) is formed in the liver from trimethylamine (TMA), a product exclusively generated by the gut microbiota from dietary phosphatidylcholine and carnitine. An alternative pathway of TMAO formation from carnitine is via the microbiota-dependent intermediate $\gamma$-butyrobetaine $(\gamma \mathrm{BB})$. Elevated TMAO levels are associated with cardiovascular disease (CVD), but little is known about TMAO in obesity. Given the proposed contribution of microbiota alterations in obesity and type 2 diabetes (T2D), we investigated the potential impact of obesity, lifestyle-induced weight loss, and bariatric surgery on plasma levels of TMAO, its microbiota-dependent intermediate $\gamma \mathrm{BB}$, and its diet-dependent precursors carnitine and choline. Methods: TMAO, $\gamma \mathrm{BB}$, carnitine, and choline were measured by high-performance liquid chromatography in 34 obese individuals (17 with and 17 without T2D) undergoing bariatric surgery and 17 controls.

Results: TMAO was not elevated in obese patients or reduced by lifestyle interventions but increased approximately twofold after bariatric surgery. Similar to TMAO, plasma levels of $\gamma B B$ were not influenced by lifestyle interventions but increased moderately after bariatric surgery. In contrast, carnitine and choline, which are abundant in nutrients, such as in red meat and eggs, and not microbiota dependent, were reduced after lifestyle interventions and rebounded after bariatric surgery.

Conclusions: The major increase in TMAO after bariatric surgery was unexpected because high TMAO levels have been linked to CVD, whereas bariatric surgery is known to reduce CVD risk. Prospective studies of gut microbiota composition and related metabolites in relation to long-term cardiovascular risk after bariatric surgery are warranted.
\end{abstract}

\section{Introduction}

$\mathrm{O}$ BESITY IS A RAPIDLY EMERGING PANDEMIC, frequently associated with the metabolic syndrome, type 2 diabetes (T2D), and cardiovascular disease (CVD), disease states that are potentially linked to chronic low-grade in- flammation. ${ }^{1}$ However, common triggering factors of obesity, T2D, and cardiovascular complications remain to be determined.

The gut microbiota, that is, the microbial inhabitants of the gastrointestinal tract, is a metabolically highly active human organ. ${ }^{2}$ Notably, alterations in the gut microbiota have been

\footnotetext{
${ }^{1}$ Section of Clinical Immunology and Infectious Diseases, Oslo University Hospital, Oslo, Norway.

${ }^{2}$ Research Institute of Internal Medicine, Oslo, Norway.

${ }^{3}$ K.G. Jebsen Centre for Inflammation Research, Oslo, Norway.

${ }^{4}$ Institute of Clinical Medicine, University of Oslo, Oslo, Norway.

${ }^{5}$ Section of Gastroenterology, Department of Transplantation Medicine, Oslo University Hospital, Oslo, Norway.

${ }^{6}$ Norwegian PSC Research Center, Oslo, Norway.

Departments of ${ }^{7}$ Surgery and ${ }^{8}$ Radiology, Nordland Hospital, Bod $\varnothing$, Norway.

${ }^{9}$ Department of Clinical Science, University of Bergen, Bergen, Norway.

${ }^{10}$ Department of Heart Disease, Haukeland University Hospital, Bergen, Norway.

${ }_{11}^{11}$ Department of Medicine, Nordland Hospital, Bodø, Norway.

${ }^{12}$ University of Troms $\varnothing$, Troms $\varnothing$, Norway.
}

(C) Marius Trøseid, et al., 2016; published by Mary Ann Liebert, Inc. This Open Access article is distributed under the terms of the Creative Commons Attribution Noncommercial License (http://creativecommons.org/licenses/by-nc/4.0/) which permits any noncommercial use, distribution, and reproduction in any medium, provided the original author(s) and the source are credited. 
associated not only with intestinal inflammation but also with conditions characterized by systemic inflammation and metabolic disturbances. Thus, several studies have suggested that the gut microbiota plays an important role in obesity, insulin resistance, and $\mathrm{T} 2 \mathrm{D}$, in part by harvesting energy from otherwise indigestible food items ${ }^{3}$ or through metabolites or toxins from the microbiota, such as lipopolysaccharide. ${ }^{4}$

Trimethylamine- $\mathrm{N}$-oxide (TMAO) is a substance formed in the liver from trimethylamine (TMA), a product exclusively generated by the gut microbiota from dietary phosphatidylcholine and carnitine, which in turn are abundant in various food sources, including eggs, dairy products, and red meat. ${ }^{5-8}$ TMAO promotes atherosclerosis, in part by enhancing the accumulation of cholesterol in foam cells, and elevated TMAO levels predict myocardial infarction, stroke, and all-cause mortality. ${ }^{5-8} \mathrm{We}$ and others have recently reported that elevated TMAO levels predict clinical outcome in heart failure patients. 9,10

An alternative pathway of TMAO formation from carnitine via the microbiota-dependent intermediate metabolite $\gamma$-butyrobetaine $(\gamma \mathrm{BB})$ was recently reported. ${ }^{11}$ Interestingly, there was an increase of atherosclerotic lesions in animals fed with $\gamma \mathrm{BB}$ compared to those fed the same diet supplemented with an antibiotic mixture, suggesting that $\gamma \mathrm{BB}$ may have a microbiota-dependent proatherogenic effect, possibly through increased TMAO formation. ${ }^{11}$

So far, few studies have investigated the potential role of TMAO in obesity or the effect of bariatric surgery. Given the proposed contribution of microbiota alterations in obesity and $\mathrm{T} 2 \mathrm{D},{ }^{12,13}$ we investigated the potential impact of obesity, lifestyle intervention, and bariatric surgery on the proatherogenic metabolite TMAO, its microbiota-dependent intermediate $\gamma \mathrm{BB}$, and its diet-dependent precursors choline and carnitine in morbidly obese patients.

\section{Materials and Methods}

\section{Patients and controls}

The study has previously been described in detail. ${ }^{4}$ In this substudy, 34 obese individuals were included, 17 with and 17 without T2D. All patients underwent conservative and subsequent surgical treatment at the regional center for treatment of morbid obesity at the Nordland Hospital, Norway. The patients were evaluated at three time points: at baseline, preoperatively (after 3 months of lifestyle intervention), and 1 year after bariatric surgery. In addition, 17 individuals with body mass index (BMI) $\leq 28 \mathrm{~kg} / \mathrm{m}^{2}$ undergoing elective laparoscopic procedures (cholecystectomy or fundoplication) were included as controls at baseline. The study was performed in accordance with the Helsinki Declaration, approved by the regional ethics committee, and written informed consent was obtained from all participants.

\section{Lifestyle interventions and dietary changes}

All patients had to undergo lifestyle changes resulting in a minimum of $10 \%$ weight loss preoperatively. The patients received repeated personal guiding by phone consultations concerning eating habits and were encouraged to perform physical activity according to the Norwegian guidelines for treatment of morbidly obese patients. ${ }^{4}$ No formal registration of diet was performed, but the patients were informed to have a total of three main meals daily and three to four small meals in between and were advised to an energy-restricted diet of $2000 \mathrm{kcal}$ for men and $1500 \mathrm{kcal}$ for women. This included a fat energy content of less than $30 \%$, complex carbohydrate energy content of less than $40 \%-50 \%$, and protein energy content up to $20 \%$. The average time from baseline to operation was 12 weeks. ${ }^{4}$

\section{Surgical methods}

Two surgical methods were used for the patients undergoing bariatric surgery: laparoscopic Roux-en-Y gastric bypass was performed by a standardized procedure and used for patients with a BMI $<50 \mathrm{~kg} / \mathrm{m}^{2}$. This procedure included a small ventricular pouch of $30 \mathrm{~mL}$ and a biliopancreatic limb of $50 \mathrm{~cm}$, with an alimentary limb of $100 \mathrm{~cm}$. For patients with a BMI $>50 \mathrm{~kg} / \mathrm{m}^{2}$, duodenal switch was performed by a standardized procedure, which included a gastric sleeve using a 32-French probe to measure the diameter, an alimentary limb of $150 \mathrm{~cm}$, and a common channel of $100 \mathrm{~cm} .{ }^{4}$ In the present study, 27 patients underwent Roux-en-Y gastric bypass, and seven underwent duodenal switch.

\section{Laboratory methods}

Fasting blood samples were collected with a standard venipuncture and stored at $-70^{\circ} \mathrm{C}$. Serum levels of $\mathrm{HbA} 1 \mathrm{c}$, creatinine, triglycerides, total cholesterol, and HDL cholesterol were analyzed in the hospital routine laboratory. Quantification of TMAO, $\gamma \mathrm{BB}$, choline, and carnitine was performed by highperformance liquid chromatography as previously described. ${ }^{10}$ Computerized tomography-based quantification of adipose tissue volumes was performed as previously described. ${ }^{4}$

\section{Statistical Analyses}

Correlations at baseline were evaluated by Pearson correlation. Differences at baseline were evaluated by independent samples $t$-test, whereas changes within subjects after lifestyle intervention and bariatric surgery were evaluated by a paired $t$-test. Skewed data were log transformed, as appropriate. A two-tailed significance level of 0.05 was used. The statistical analyses were performed with SPSS software, version 19.0 (SPSS, Inc., Chicago, IL).

\section{Results}

\section{No elevation of TMAO or its precursors in obese subjects}

The baseline characteristics of the patients and controls are given in Table 1. There were no significant differences in plasma levels of TMAO $(P=0.16), \gamma \mathrm{BB} \quad(P=0.93)$, choline $(P=0.82)$, or carnitine $(P=0.27)$ between obese subjects and controls (Fig. 1) or between diabetic and nondiabetic obese subjects $(P=0.75, P=0.33, P=0.25$, and $P=0.13$, respectively).

\section{Choline, carnitine, and $\gamma B B$, but not TMAO, are associated with visceral adipose tissue}

Choline, carnitine, and $\gamma \mathrm{BB}$, but not TMAO, correlated significantly with visceral adipose tissue $(r=0.65, P=0.006$; $r=0.51, P=0.044$; and $r=0.75, P=0.001$, respectively) but not with other cardiometabolic risk factors listed in Table 1, including BMI, lipids, and diabetes status. Furthermore, carnitine and $\gamma \mathrm{BB}$ correlated negatively with subcutaneous 
Table 1. Baseline Characteristics of Obese SUBJECTS AND CONTROLS

\begin{tabular}{lcc}
\hline & Obese $(\mathrm{n}=34)$ & Controls $(\mathrm{n}=17)$ \\
\hline Gender (f:m, $n)$ & $19: 15$ & 12.5 \\
Age (years) & $43.2(9.0)$ & $48.1(14.9)$ \\
Smoking $(n /$ total $)$ & $6 / 34$ & $2 / 17$ \\
Diabetes $(n /$ total $)$ & $17 / 34$ & $1 / 17$ \\
BMI $\left(\mathrm{kg} / \mathrm{m}^{2}\right)$ & $44.8(6.9)$ & $25.3(2.1)$ \\
Cholesterol $(\mathrm{mM})$ & $5.18(1.08)$ & $5.53(0.93)$ \\
Triglycerides $(\mathrm{mM})$ & $1.78(0.87)$ & $1.05(0.30)$ \\
HbAlc $(\%, \mathrm{mM})$ & $6.57(1.15)$ & $5.55(0.21)$ \\
\hline
\end{tabular}

Data presented as mean (standard deviation) or number $(n)$. BMI, body mass index.

adipose tissue $(r=-0.52, P=0.040$; and $r=-0.63, P=0.009$, respectively).

\section{Diet-dependent metabolites choline and carnitine are reduced after lifestyle interventions}

Choline levels were significantly reduced after lifestyle interventions from 8.5 [95\% CI 7.2-9.7] to 6.6 [5.7-7.5] $\mu \mathrm{M}$ $(P<0.001)$ but rebounded partly after bariatric surgery to $7.1[6.1-8.1] \mu \mathrm{M}(P=0.035)$ (Fig. 1A). Plasma levels of carnitine followed approximately the same pattern, with a significant reduction after lifestyle interventions from 37.8 [34.4-41.2] to 34.4 [31.1-37.6] $\mu \mathrm{M}(P=0.009)$ but returned to baseline levels after bariatric surgery (Fig. 1B).

\section{Microbiota-dependent metabolites TMAO and $\gamma B B$ increase after bariatric surgery}

Regarding TMAO and $\gamma \mathrm{BB}$, there was no effect of lifestyle intervention. However, 1 year after bariatric surgery, TMAO plasma levels more than doubled $(10.5[7.5-13.5] \mu \mathrm{M})$ compared to preoperative $(4.4[2.8-6.0] \mu \mathrm{M}, P<0.001)$ and baseline (4.7 [3.7-5.8] $\mu \mathrm{M}, P<0.001)$ levels (Fig. 1C). The same pattern was seen regardless of surgical method, with six of seven patients undergoing duodenal switch and 23 of 27 undergoing Roux-en-Y gastric bypass increasing in TMAO levels 1 year after bariatric surgery compared to preoperative levels (paired test, $P<0.001$, and $P=0.004$, respectively) and admission ( $P=0.004$ for both types of surgery). Moreover, the same pattern was seen regardless of gender, with 14 of 19 women and 12 of 15 men increasing in TMAO.

Similar to TMAO, $\gamma \mathrm{BB}$ levels increased significantly after bariatric surgery $(P=0.005$ vs. preoperative levels, $P=0.007$ vs. baseline levels) (Fig. 1D). However, the relative increase in $\gamma \mathrm{BB}$ levels was $\sim 10 \%$ compared with an approximate twofold increase in TMAO. Notably, $\gamma \mathrm{BB}$ and TMAO levels did not correlate significantly at any time points.

We have previously reported a significant decrease in CRP levels 1 year after bariatric surgery in the total study cohort. ${ }^{14}$ As TMAO has been proposed to trigger macrophage inflammation, we correlated changes in TMAO with those in CRP from preoperative levels to 1 year after surgery; however, no significant correlation was found $(r=-0.11, P=0.55)$.

Furthermore, as TMAO is eliminated by urinary excretion and has been reported to be increased in patients with renal dysfunction, ${ }^{15}$ we measured creatinine levels to exclude the possibility of decreased renal function causing reduced TMAO secretion after bariatric surgery. In contrast, creatinine levels were moderately reduced after bariatric surgery, from a preoperative level of 81 [59-104] to 76 [52-100] $\mathrm{mM} 1$ year after bariatric surgery $(P=0.037$ vs. preoperative and baseline levels).

Finally, as half of the obese subjects had diabetes, and TMAO has been shown to strongly predict clinical endpoints in type 2 diabetes, ${ }^{16,17}$ we correlated changes in
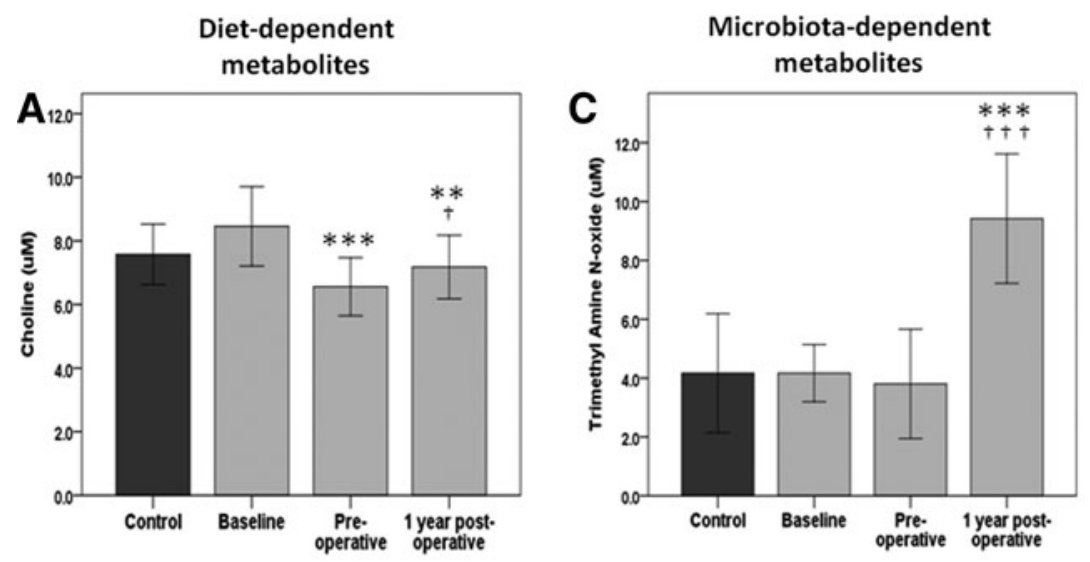

FIG. 1. Plasma levels of choline (A), carnitine (B), trimethylamine- $\mathrm{N}$-oxide (TMAO) (C), and $\gamma$-butyrobetaine (D) in controls (dark gray bars) and morbidly obese subjects (light gray bars) at baseline, after lifestyle intervention (preoperative), and 1 year after surgery (postoperative). Data presented as mean $(95 \% \mathrm{CI})$.
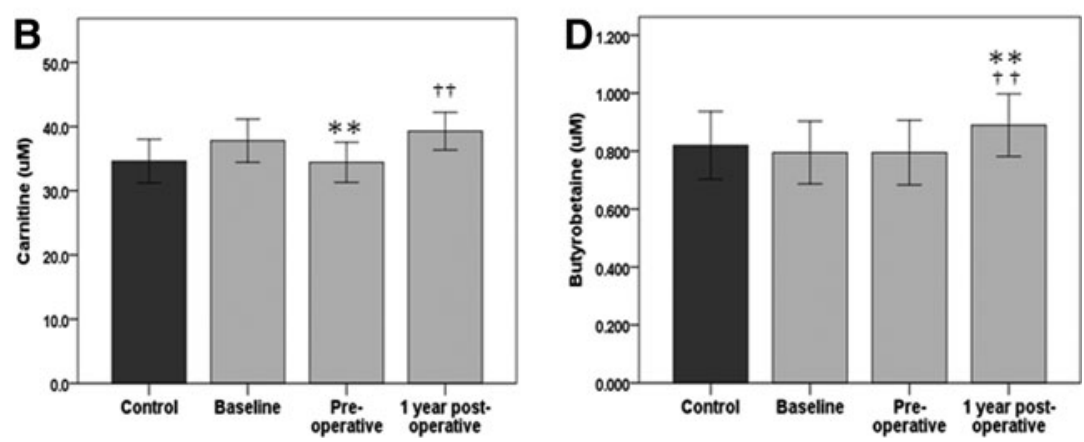
$* * * P<0.001, * * P<0.01$, versus baseline; ${ }^{\dagger \dagger} P<0.001,{ }^{\dagger \dagger} P<0.01,{ }^{\dagger} P<0.05$ versus preoperative. 
TMAO with those in HbA1c. Levels of HbA1c decreased 1 year after bariatric surgery to 5.74 [5.55-5.93]\% compared to preoperative levels of $6.57[6.18-7.00] \%(P<0.001)$. Interestingly, the increase in TMAO was significantly correlated with the corresponding decrease in $\mathrm{HbAlc}$ from preoperative levels to 1 year after bariatric surgery $(r=-0.39$, $P=0.025)$.

\section{Discussion}

The increase in levels of the proatherogenic metabolite TMAO after bariatric surgery was substantial and unexpected, representing a major contrast to the known beneficial effects of this intervention on metabolic parameters. One explanation could be adaptive shifts in the gut microbiota with increased ability to metabolize dietary choline and carnitine to TMAO precursors. The corresponding increase in $\gamma \mathrm{BB}$ is in support of this explanation, although the increase was of a much smaller magnitude.

While only antibiotics and diet interventions so far have been reported to alter the TMAO levels in humans, ${ }^{7,18}$ the findings in the present study are in line with increased TMAO levels observed in rats undergoing Roux-en-Y gastric bypass. ${ }^{19}$ This was suggested to be caused by postsurgery starvation caused by caloric restriction, ${ }^{19}$ but increases of plasma carnitine and choline postsurgery in our patients make this a less likely explanation. Rather, several recent studies suggest that TMAO elevation postsurgery is caused by gut microbiota alterations.

A recent work by Tremaroli et al. reported altered gut microbiota composition and function with corresponding elevation of TMAO levels in women 9 years after undergoing bariatric surgery compared with obese controls. ${ }^{20}$ Our findings confirm and expand these results, finding increased TMAO levels in both men and women after bariatric surgery compared with preoperative levels. To the best of our knowledge, our work is the first longitudinal study to investigate TMAO levels before and after bariatric surgery. Furthermore, and in line with our results, levels of choline were not altered by bariatric surgery. Moreover, the elevations of TMAO levels in the Tremaroli study were only seen in women undergoing Roux-en-Y gastric bypass and not in those operated by vertical banded gastroplasty, which reduces the stomach without bypassing the small intestine. ${ }^{20}$ In our study, the same pattern was seen in patients undergoing Roux-en-Y and duodenal switch, both procedures that induce malabsorption and bypass parts of the small intestine, although we are not aware of studies directly comparing Roux-en-Y gastric bypass and duodenal switch regarding gut microbiota composition.

In contrast to its precursors choline, carnitine, and $\gamma \mathrm{BB}$, we found that TMAO levels were not associated with visceral adipose tissue. Furthermore, none of the metabolites were elevated in obese subjects or associated with BMI. In the study by Tremaroli et al., gut microbiota changes were independent of BMI, suggesting that gut microbiota changes result from bariatric surgery and not by changes in BMI per $s e .^{20}$ One previous study reported major microbiota alterations associated with improved metabolic status and reduced inflammation after bariatric surgery. ${ }^{21}$ In contrast, another study reported reduction in Eubacterium, which has been associated with protection against carotid atherosclerosis, and increased relative abundance of Enterobacter, ${ }^{22}$ comprising multiple common pathogens, such as E. coli and Pseudomo- nas. In the work by Tremaroli et al., it was suggested that the increased TMAO levels after bariatric surgery could occur due to shortening of the small bowel and less anaerobic metabolism by the gut microbiota. Furthermore, increased levels of microbes, such as E. coli, which could use TMAO as a terminal electron acceptor, and Pseudomonas, which in theory can convert TMA to TMAO by the enzyme TMA monooxidase, could contribute to increased TMAO levels. ${ }^{20}$

Whereas choline and carnitine may be converted directly to TMA via bacterial enzyme systems, typically in the colon, an alternative bacterial conversion of carnitine to $\gamma \mathrm{BB}$ was recently reported to occur in the small intestine, with $\gamma \mathrm{BB}$ further partly converted to TMA in the colon. ${ }^{11}$ Overall, the approximately twofold increase in TMAO compared to a $10 \%$ increase in $\gamma \mathrm{BB}$ could imply major shifts occurring in the large bowel microbiota, but bariatric surgery-induced alterations in the small bowel could also contribute.

Several additional factors could contribute to the increased TMAO levels after bariatric surgery. First, in contrast to $\gamma \mathrm{BB}$, TMAO formation is dependent on oxidation by hepatic flavin-containing mono-oxygenase (FMO3), and reduced hepatic steatosis and induced hepatic TMAO formation after bariatric surgery could also play a role. Furthermore, FMO3 is suppressed by insulin in vitro, ${ }^{17}$ and it could be speculated that improved insulin sensitivity and lower insulin levels after bariatric surgery could contribute to increased FMO3 expression and hence increased TMAO production, supported by our finding of increased TMAO levels being negatively correlated with reduced $\mathrm{HbA1c}$.

Moreover, TMAO is eliminated by urinary excretion and increased in patients with renal dysfunction, ${ }^{15}$ although we did not see any reduction in renal function after surgery. Finally, dietary changes occurring after surgery could contribute, supported by an increase in carnitine and choline levels after initial diet-induced reduction. Carnitine and choline did, however, not increase beyond baseline values (while TMAO increased twofold), pointing to altered microbial metabolism as a key element.

This study has several strengths. It is the first longitudinal study to examine TMAO levels in the same subjects before and after bariatric surgery, providing strong proof of a real increase in TMAO after surgery. There was also a structured lifestyle intervention program before surgery with a requirement of $10 \%$ weight loss, providing the opportunity for studying effects of nonsurgical weight loss. However, we do not have any information about dietary changes or gut microbiota changes occurring in the study subjects after bariatric surgery, which are significant limitations of the study. Furthermore, it would have strengthened the study if the controls were also followed longitudinally to detect potential time-dependent effect on the biomarkers studied. However, it is difficult to find suitable explanations for timedependent effects on the microbiome in a group of individuals not undergoing structured changes in diet or other changes in lifestyle or other environmental factors. Moreover, the small sample size increases the risk of type II statistical errors. However, type I errors are less likely, and the marked increase in TMAO levels 1 year after bariatric surgery is most likely correct.

In conclusion, the major increase in TMAO after bariatric surgery was unexpected because high TMAO levels have been linked to CVD, whereas bariatric surgery is known to reduce CVD risk. ${ }^{23,24}$ Prospective studies are therefore 
warranted to characterize the gut microbiota profile and microbiota-related metabolites in relation to long-term cardiovascular risk after bariatric surgery.

\section{Author Disclosure Statement}

No competing financial interests exist.

\section{References}

1. Pradhan A. Obesity, metabolic syndrome, and type 2 diabetes: Inflammatory basis of glucose metabolic disorders. Nutr Rev 2007;65:S152-S156.

2. Tremaroli V, Backhed F. Functional interactions between the gut microbiota and host metabolism. Nature 2012;489: 242-249.

3. Burcelin R, Serino M, Chabo C, et al. Gut microbiota and diabetes: From pathogenesis to therapeutic perspective. Acta Diabetol 2011;48:257-273.

4. Troseid M, Nestvold TK, Rudi K, et al. Plasma lipopolysaccharide is closely associated with glycemic control and abdominal obesity: Evidence from bariatric surgery. Diabetes Care 2013;36:3627-3632.

5. Backhed F. Meat-metabolizing bacteria in atherosclerosis. Nat Med 2013;19:533-534.

6. Koeth RA, Wang Z, Levison BS, et al. Intestinal microbiota metabolism of L-carnitine, a nutrient in red meat, promotes atherosclerosis. Nat Med 2013;19:576-585.

7. Tang WH, Wang Z, Levison BS, et al. Intestinal microbial metabolism of phosphatidylcholine and cardiovascular risk. N Engl J Med 2013;368:1575-1584.

8. Wang Z, Klipfell E, Bennett BJ, et al. Gut flora metabolism of phosphatidylcholine promotes cardiovascular disease. Nature 2011;472:57-63.

9. Tang WH, Wang Z, Fan Y, et al. Prognostic value of elevated levels of intestinal microbe-generated metabolite trimethylamine-N-oxide in patients with heart failure: Refining the gut hypothesis. J Am Coll Cardiol 2014;64:19081914.

10. Troseid M, Ueland T, Hov JR, et al. Microbiota-dependent metabolite trimethylamine- $\mathrm{N}$-oxide is associated with disease severity and survival of patients with chronic heart failure. J Intern Med 2015;277:717-726.

11. Koeth RA, Levison BS, Culley MK, et al. gammaButyrobetaine is a proatherogenic intermediate in gut microbial metabolism of L-carnitine to TMAO. Cell Metab 2014;20:799-812.

12. Karlsson FH, Tremaroli V, Nookaew I, et al. Gut metagenome in European women with normal, impaired and diabetic glucose control. Nature 2013;498:99-103.

13. Qin J, Li Y, Cai Z, et al. A metagenome-wide association study of gut microbiota in type 2 diabetes. Nature 2012;490: 55-60.
14. Nestvold TK, Nielsen EW, Ludviksen JK, et al. Lifestyle changes followed by bariatric surgery lower inflammatory markers and the cardiovascular risk factors C3 and C4. Metab Syndr Relat Disord 2015;13:29-35.

15. Tang WH, Wang Z, Kennedy DJ, et al. Gut microbiotadependent trimethylamine N-oxide (TMAO) pathway contributes to both development of renal insufficiency and mortality risk in chronic kidney disease. Circ Res 2015;116: 448-455.

16. Lever M, George PM, Slow S, et al. Betaine and trimethylamine-N-oxide as predictors of cardiovascular outcomes show different patterns in diabetes mellitus: An observational study. PLoS One 2014;9:e114969.

17. Miao J, Ling AV, Manthena PV, et al. Flavin-containing monooxygenase 3 as a potential player in diabetesassociated atherosclerosis. Nat Commun 2015;6:6498.

18. Stella C, Beckwith-Hall B, Cloarec O, et al. Susceptibility of human metabolic phenotypes to dietary modulation. $J$ Proteome Res 2006;5:2780-2788.

19. Ashrafian H, Li JV, Spagou K, et al. Bariatric surgery modulates circulating and cardiac metabolites. J Proteome Res 2014;13:570-580.

20. Tremaroli V, Karlsson F, Werling M, et al. Roux-en-Y gastric bypass and vertical banded gastroplasty induce long-term changes on the human gut microbiome contributing to fat mass regulation. Cell Metab 2015;22:228-238.

21. Graessler J, Qin Y, Zhong H, et al. Metagenomic sequencing of the human gut microbiome before and after bariatric surgery in obese patients with type 2 diabetes: Correlation with inflammatory and metabolic parameters. Pharmacogenomics J 2013;13:514-522.

22. Karlsson FH, Fak F, Nookaew I, et al. Symptomatic atherosclerosis is associated with an altered gut metagenome. Nat Commun 2012;3:1245.

23. Sjostrom L, Narbro K, Sjostrom CD, et al. Effects of bariatric surgery on mortality in Swedish obese subjects. $N$ Engl J Med 2007;357:741-752.

24. Sjostrom L, Peltonen M, Jacobson P, et al. Bariatric surgery and long-term cardiovascular events. JAMA 2012;307: 56-65.

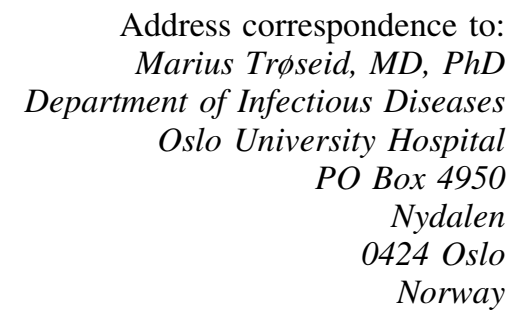

E-mail: troseid@hotmail.com 